\title{
POD investigation of the unsteady turbulent boundary layer developing over porous moving flexible fishing net structure
}

\author{
Philippe Druault ${ }^{1,{ }^{*}}$, Elkhadim Bouhoubeiny ${ }^{1}$ and Grégory Germain ${ }^{2}$
}

${ }^{1}$ Institut Jean Le Rond d'Alembert, University Pierre and Marie Curie-Paris 6, CNRS UMR 7190, case 162, 75252 Paris Cedex 5, France

${ }^{2}$ IFREMER, Hydrodynamic and Metocean Service, 150 Quai Gambetta, 62321 Boulogne-sur-Mer, France

*: Corresponding author : P. Druault, email address : philippe.druault@upmc.fr

\begin{abstract}
:
Particle image velocimetry (PIV) measurements are made to investigate the boundary layer developing over a modeled bottom trawl. The random motion of the fishing net structure as well as the flexibility and the porosity of this structure means that it is not enable to access the main characteristics of such a flow, using classical post-processing mathematical tools. An innovative posttreatment tool based on proper orthogonal decomposition (POD) is then developed to extract the mean velocity flow field from each available PIV instantaneous unsteady velocity field. In order to do so, the whole available velocity database is used to compute POD eigenfunctions and the first POD modes are identified as representing the mean flow field. It is then possible to deduce the mean boundary layer flow field for each position of the fishing net structure during PIV measurements. It is then observed that the mean flow field strongly depends on multiple parameters such as surface curvature, structure porosity, random motion of the structure. Streamwise evolution of classical thicknesses of boundary layer flow are also analyzed. The present work also provides benchmark PIV data of the unsteady flow developing on fishing net porous structures, which helps the progress in unsteady numerical codes for this investigation.
\end{abstract}

\section{Introduction}

The investigation of hydrodynamic flow developing around fishing gear is of great importance not only for commercial fishing operations including the fishing vessel energy efficiency but also for the biological and socio-economical environment. For instance, such an investigation is useful in the interpretation of fish behavior in trawls and in the understanding of the selectivity process (the ability of fishing gears to prevent non-target fish catches). Very few studies have been carried out previously. Paschen et al. (2008) investigated the fluid net structure interaction of a trawl and specifically they focused on how the net structure affects the current. Recently, Paschen (2009) theoretically analyzed the effect of a fluid blockage on velocity distribution inside and on the load of a netting construction. $\mathrm{He}$ finally stated that this fluid-structure interaction in pelagic trawls has probable consequences for the selectivity of the fishing gear. Pichot et al. (2009) considered a rigid cod-end structure with a closed and an open net entrance. They used Laser Doppler Velocimetry (LDV) measurements to investigate the mean flow field around this fixed cod-end structure. The hydrodynamic flow of fishing gear is more complex due to the unsteady flow interacting with the free moving fishing net structure and today remains elusive. It is unquestionably different from that over a rigid model even if some similarities were observed in the near wake of the structure (Bouhoubeiny et al. 2011). Indeed, based on Particle image velocimetry (PIV) measurements, Bouhoubeiny et al. (2011) studied the flow past a modeled bottom trawl free to move. It was then observed that the frequencies associated with the oscillatory motion of the moving 
trawl correspond to those detected in the near wake flow. Moreover, the highly three-dimensional flow around the fishing net structure is very complex which makes numerical simulations quite difficult (Meyler, 2008). Indeed, one particular difficulty concerns the effect of the flow on the net structure supporting deformation, flexibility, oscillations. Other previous numerical studies have focused on the computation of the whole drag force of a fishing structure. This becomes a key issue for researchers and fishermen due to its impact on fuel consumption. Basically, the computation of drag force has been carried out by using empirical formulations using the towing velocity as reference water velocity (Priour et al, 2009). As a consequence, considering that the drag is a direct function of the towing speed, many fishermen limit this velocity in order to reduce fuel consumption. However, such a choice may be questionable and it is still subject to uncertainties due to the lack of data information about the velocity field in the close vicinity of the fishing net structure. Finally, the behaviour of a fishing net structure moving relative to a fluid cannot be accurately described without an understanding of the boundary layer development on net parts at low incidence with the flow.

A better knowledge of the boundary layer flow is of major interest for multiple applications such as the determination of the fluid passage through the net structure or the numerical modeling of the fishing net structure. But such a flow encompasses several of the most complex phenomena in classical Turbulent Boundary Layer (TBL) flows, such as i) porosity of the structure, ii) highly deformable structure (flexibility of the net), iii) random unsteady motion of the structure, iv) curvature effects; v) roughness of the fishing net surface (presence of twines and knots).

Basically, two- and three-dimensional spatially developing TBL on a flat plate are encountered in numerous fluid engineering applications. They have been intensely studied in the past, theoretically, experimentally as well as numerically (Schlichting and Gersten, 2000). For instance, the effect on roughness of the wall surface on the boundary layer flow properties has been considerably investigated (Jimenez, 2004). Boundary layer flows developing over bodies with concave or convex curvature which can be similar to those developing on fixed wavy surfaces have been also previously analyzed for particular applications (Shen et al., 2003 and references therein). The development of TBL on a permeable surface has been also previously examined to elucidate the transport process through a porous structure. Injection or withdrawal of fluid through a porous wall has been studied for practical engineering applications related to TBL control strategies such as drag reduction. But none of them have been cumulated at the same time. In fact, the effect of the porosity of a fishing net structure is quite different to previous applications due to the free movement and the instantaneously changeable curvature of the structure. Due to the great interaction between each of these parameters, the whole resulting TBL flow can not be regarded as a combination of separately individual parameters. The fluid passage through the porous fishing net structure surface, coupled with the random motion of the structure, can significantly modify the flow field.

The first key issue concerns the entire source of unsteadiness present in TBL developing over the fishing net flexible structure. Indeed, relatively little is known about the interaction between time-dependent surface deformation and boundary layer development. Even if unsteady TBL flows occur in many practical situations such as turbomachinery, helicopter blades, animal propulsion, etc., the response of TBL flows to unsteadiness remains effectively questionable. Apart from the porosity of the fishing net structure, the present TBL flow over a flexible moving structure can be related to TBL developing on unsteady undulatory fish (e.g. Carpenter et al., 2000; Anderson et al., 2001) or TBL flow over a two-dimensional flexible wall (Brereton, 1989, Kunze and Brücker, 2011). The characterization of TBL developing on swimming fish has been investigated with PIV measurements by Anderson et al. (2001). They concluded that the motions of the body is favorable in that it leads to laminarize the flow inducing a drag reduction phenomena. Finally, it is essential to predict precisely the boundary layer development over the fish's body not only to enhance the understanding of the drag reduction mechanism but also for its influence on hydrodynamic receptors located on this body (McHenry et al., 2008).

When dealing with fully unsteady flows around a randomly oscillating structure, a first difficulty concerns the determination of the mean flow field. To implement the classical time averaged operator, it is necessary to have a sufficient number of velocity samples available on the same grid mesh taking into account the movement of the structure. A first solution may consist in using the body-structure frame of reference (Anderson et al., 2001, Epps and Techet, 2010). When dealing with a known body-oscillation motion, the mean flow field can be achieved by using the phase average operator (Druault et al., 2005, Kunze and Brücker, 2011). Indeed, when the body has periodic oscillation cycles, the phase average operator can be used to detect each measure at the same phase in accordance with a reference oscillator. Both of these solutions are inappropriate in the present work. The structure-oscillations are fully random that instantaneously modifies the porosity and the curvature of the fishing structure. That avoids any use of a phase average operator in this context. Finally, to access the TBL flow characteristics including the mean flow field, it is necessary to implement new post-processing tool aimed at determining the mean flow field for each available instantaneous velocity field. 
The main goal of the present work is therefore to propose a new mathematical procedure allowing the extraction of the mean flow from a TBL flow having multiple sources of unsteadinesses. For such a purpose, an expansion of the Proper Orthogonal Decomposition (POD) procedure is proposed. Once this step achieved, preliminary investigations of the mean flow characteristics of the resulting TBL flow are provided.

The experimental facility and the measurements are described in $\S 2$. In section 3 , the POD mathematical concept is recalled. A detailed analysis of the resulting POD eigenfunctions and coefficients is provided in $\S 4$. Then, applying the POD technique to an experimental database, the mean streamwise velocity profiles as well as the streamwise evolution of the boundary layer thicknesses are successively shown in $\S 5$.

\section{Experimental apparatus and PIV measurements}

\subsection{Flume tank and fishing net structure}

Experimental studies are carried out in the IFREMER (French Institute for the Exploitation of the Sea) wave and current circulation flume tank displayed in Figure 1. Dimensions of the flume tank are $18 \mathrm{~m}$ long by $4 \mathrm{~m}$ wide and $2 \mathrm{~m}$ deep. A side observation window of $8 \mathrm{~m}$ by $2 \mathrm{~m}$ placed on one side of the tank allows users to observe the behaviour of the models during trials. The bottom of the flume tank is a conveyor belt which can be synchronized with the water speed in order to simulate devices in contact with the bottom, like bottom trawls. The turbulence levels can be adjusted between $5 \%$ and $28 \%$ (by the modification of grids and flow straighteners at the entry of the test section) in order to simulate more realistic flows. The flow streamwise velocity can be specified in the following range $[0.1 ; 2.2]$ $\mathrm{m} . \mathrm{s}^{-1}$.

The fishing net structure is a 1:10 scaled model of a commercial bottom trawl (Figure 2). The model located at the bottom of the flume tank is free to move in the water flow. For such a configuration the conveyor belt is synchronized with the flow speed. The model is $4 \mathrm{~m}$ long with a swept width of $1.8 \mathrm{~m}$ and a swept height of $0.45 \mathrm{~m}$ (Figure 2). The model is made with diamond-shaped meshes of $8 \mathrm{~mm}$ long (the length of the meshes between two knots), with a twine diameter of $0.6 \mathrm{~mm}$. In this work, measurements are made with a fixed input streamwise velocity of $U_{\infty}=0.45 \mathrm{~m} . \mathrm{s}^{-1}$ and a turbulence intensity rate of $5 \%$. This turbulence level corresponds to the one computed in the far field where the flow is uniform. For such a computation, we have considered the upper zone of the first PIV measurement plane detailed below. Using Reynolds decomposition, the turbulence level has been determined and it is of an order of $4.8 \%$. In this flume tank, we voluntary specify such turbulence level to partly reproduce the one existing in oceans or seas. Moreover, such turbulence level acts as an amplification rate of the porous structure oscillations but has no consequence on the proposed method and results.

To investigate the TBL development over such structure, measurements are made upstream of the bottom trawl that is just at the beginning of the porous structure. The corresponding Reynolds numbers (based on the upstream velocity) are 264 if it is based on the twine diameter, 3520 if it is based on the diamond-shaped meshes and $10^{5}$ if it is based on the cod-end diameter or the vertical opening of the trawl. These Reynolds numbers highlight the difficulty in characterizing the flow around this kind of device due to different characteristic parameters of the structure, its porosity and its permanent motion.

\subsection{PIV measurement system}

In this study, two-dimensional Time-Resolved PIV measurements are carried out. Illumination is provided by a standard, frequency-doubled, double-cavity Nd:YAG laser (NewWave, Gemini PIV) with a pulse energy of up to $120 \mathrm{~mJ}$ per pulse at $532 \mathrm{~nm}$, and maximum repetition of $15 \mathrm{~Hz}$. The seeding particles used for the experiments are silver-plated glass spheres of $15 \mu \mathrm{m}$ diameter. On the recording side, a high resolution CCD camera with a $20 \mathrm{~mm}$ focal lens is used. The physical area of approximately $0.38 \mathrm{~m}$ by $0.28 \mathrm{~m}$ is imaged onto 1600 by 1280 pixels $^{2}$. This camera has an acquisition rate of $15 \mathrm{~Hz}$ that allows the investigation of the frequencies associated with the free motion of the fishing net structure (Bouhoubeiny et al., 2011). The Flow Map 1500 software from Dantec dynamics is used for PIV image processing. Finally, instantaneous velocity fields are obtained using a cross-correlation PIV algorithm. PIV system is implemented to determine the longitudinal $u$ (or $u_{1}$ ) and transverse $v$ (or $u_{2}$ ) velocity components along the $x$ and $y$ directions respectively. For the calculation of the velocity vectors, the PIV recordings are correlated in overlapping windows of $32 \times 32 \mathrm{pixel}^{2}$, and adjacent windows are $25 \%$ overlapped. This yields $199 \times 74$ velocity vectors on a regular mesh of $383 \times 143 \mathrm{~mm}^{2}$. The physical discretization corresponds to a resolution down to $1.9 \mathrm{~mm}$ in each direction and the time separation between two PIV pictures is $0.066 \mathrm{~s}$. 


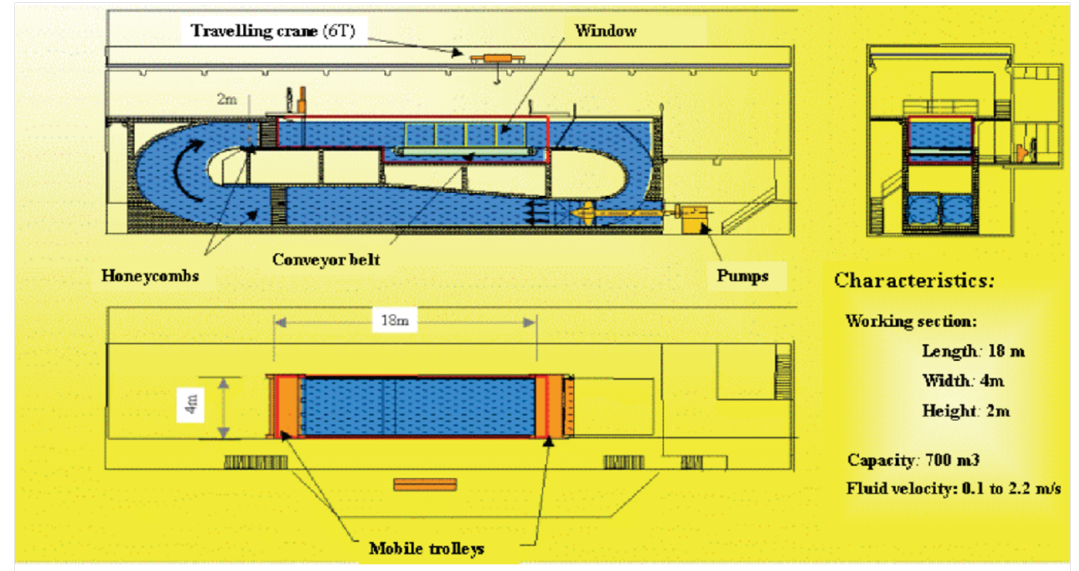

Fig. 1 Schematic representation of IFREMER flume tank.
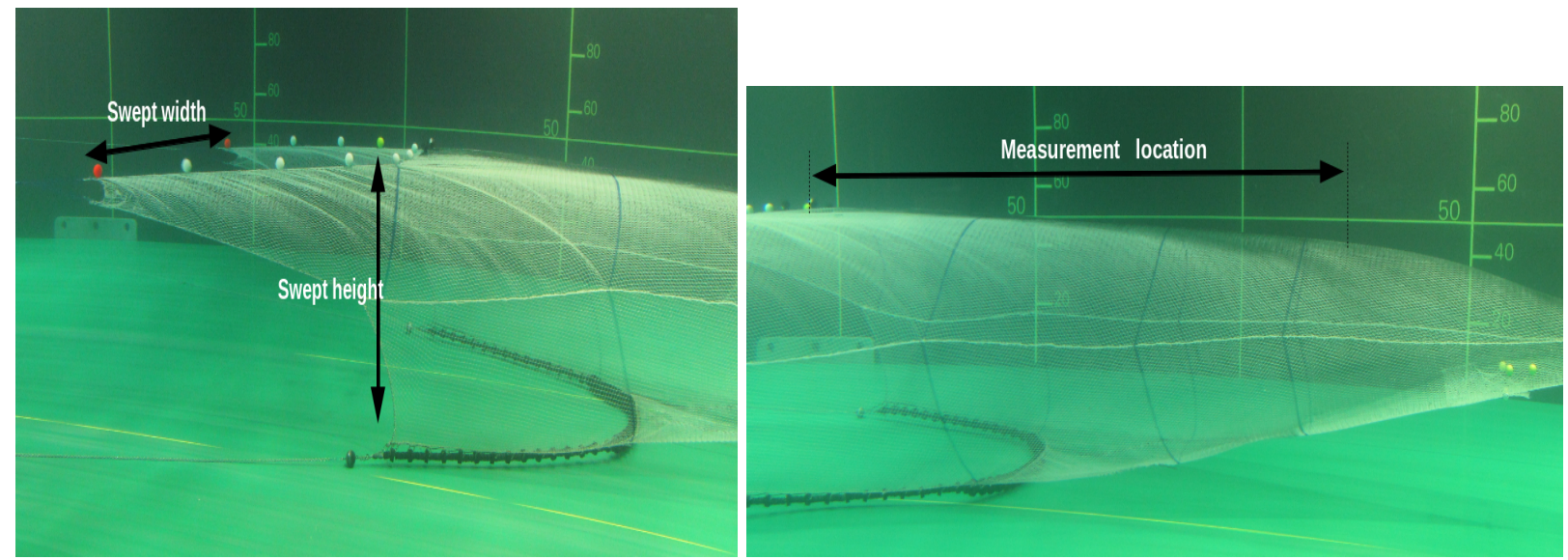

Fig. 2 Scaled bottom trawl used to investigate the boundary layer. PIV measurement span on the horizontal part of the bottom trawl. Note that the measurement scale present in background wall has no connection with the present work.

The flow configuration corresponds to the turbulent flow around the moving bottom trawl described above. To investigate the TBL developing over the trawl located at the bottom of the flume tank, the laser is placed above the bottom trawl and the camera is outside. Data acquisition begins after several fishing net structure oscillations. 500 $\left(=N_{t}\right)$ instantaneous velocity fields are measured in 5 different planes along the bottom trawl. Figure 3 indicates the locations of these planes represented alternatively with a dashed blue line and a blue line. Note that two consecutive PIV measurement planes have an overlapping region. On this figure, red and black dashed lines indicate the bottom and top positions of the trawl respectively, during PIV measurements. Note that in this figure, $Y$-axis origin $(y=0)$ does not correspond to any physical position of the bottom trawl. Conversely, $X$-axis values are specified in agreement with the beginning of the fishing net structure that corresponds to $x=0$.

Each illuminated plane is initially retained to be perpendicular to the porous structure. Note that during measurements the measurement plane may not remain exactly perpendicular to the mesh due to the flexibility of the structure. It is expected that such variations can be neglected. They have little effect on the proposed method.

\subsection{Investigation of the fishing net structure free oscillation}

The random motion of the structure is the result of unsteady hydrodynamic forces and of the flexibility of the net. Evaluating the random motion of the fishing net structure within the field of view requires first to locate the trawl surface in the data image. Recently, based on PIV measurements around a freely swimming fish, Siddiqui (2007) proposed a numerical scheme based on the extraction of bright and dark regions to isolate the moving body. Nevertheless, such a procedure can not be applied in the present study especially due to the porosity of the structure. 


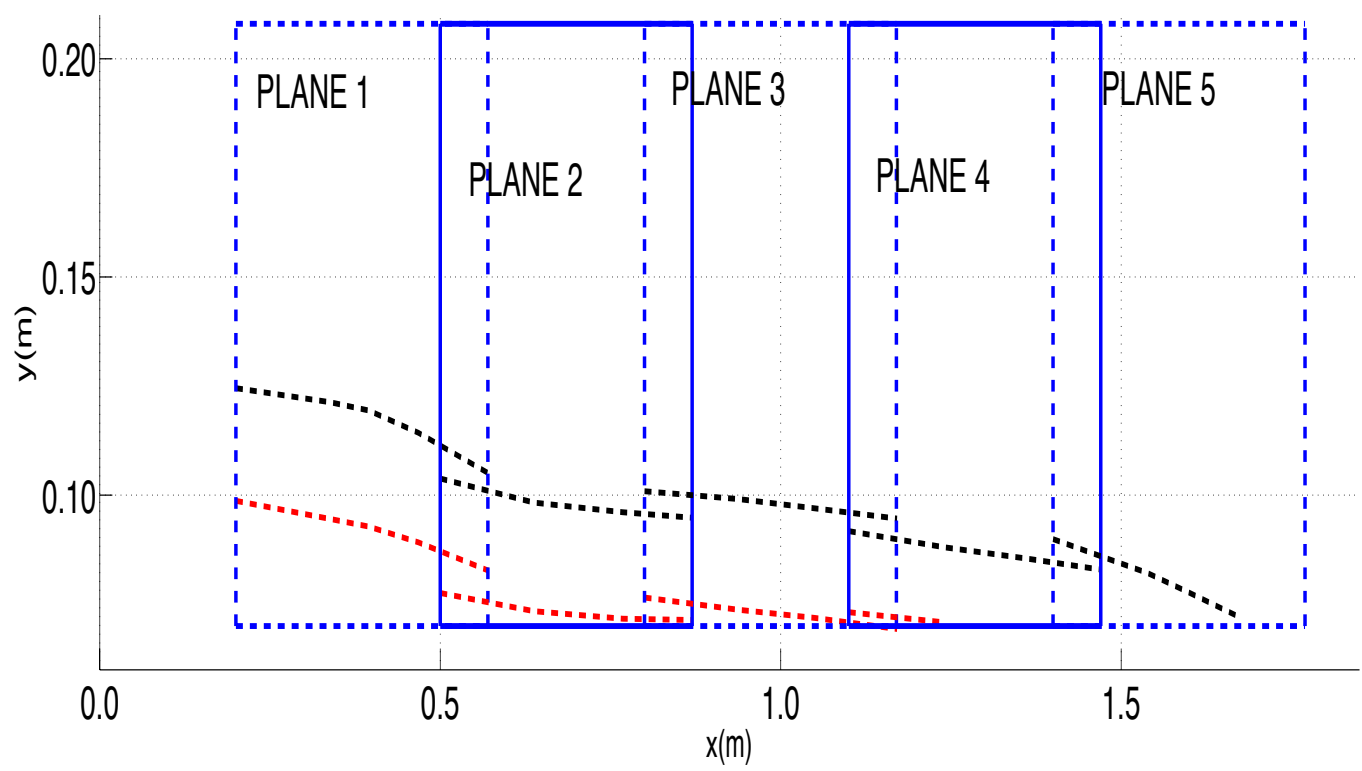

Fig. 3 Overlapped PIV measurement planes (blue lines). Locations of the top and bottom positions of the moving structure in each measurement plane are indicated with black and red lines respectively.

After, the fishing net structure is identified manually in each frame. For such a purpose, a similar procedure is followed. In each PIV picture, four points located on the surface of the bottom trawl are extracted and marked. The fishing structure moves quite slowly, and the local fishing net deformation is quite small compared to the amplitude of the fishing structure oscillations. Thus, the time evolution of each marked point is quite similar in a same PIV plane justifying that such evolution can be associated with the time evolution of the whole fishing net structure. As an illustration, figure 4 displays the time evolution of one point extracted from the trawl surface in each PIV measurement plane. The corresponding spectral representation is also given in this figure. Although the instantaneous bottom trawl motion is quite different in each plane, the spectral representation exhibits a similar frequency peak around $f=0.03 \mathrm{~Hz}$. In each plane, the magnitude of the movements ranges in value from $-0.01 \mathrm{~m}$ to $0.01 \mathrm{~m}$ around the mean average value. However, these instantaneous motions reveal large discrepancies. This can be related to several parameters. Firstly, in these figures, the time evolution of the porous structure is only provided along the transverse direction. Even if the fishing net structure surface oscillates mainly in the field of view of the particle-imaging camera because of the transverse motion of the body, it also moves in the other planes due to the net flexibility and the high-fully three dimensional unsteady forces acting on trawl surface. This out-of-plane motion can not be visualized from current $2 \mathrm{D}$ visualizations. Secondly, the upper surface of the trawl is quite different in each plane that is the curve is more pronounced in the PIV measurement planes 4 and 5 than in the other ones. Globally, the transverse curvature and flexibility of the net structure strongly affect the resulting surface parameters.

In figure 4, three moments are marked in each plane. These points correspond to some particular positions of the moving fishing net structure. A black circle indicates the highest $y$-position of the trawl during PIV measurements. A black box and a black star are associated with a similar location (approximately the time average value of the movement) but correspond to a upward trawl motion and a backward trawl motion respectively. These 3 instants will be used in the next sections in order to preliminarily observe the influence of the trawl motion on the TBL flow characteristics.

\section{Mathematical post-processing tool: Proper Orthogonal Decomposition (POD)}

\subsection{Brief mathematical procedure}

The proper orthogonal decomposition (POD) is a powerful and interesting method for data analysis. A particularly interesting application to turbulent flow is its ability to provide a low-dimensional description of turbulent flows by 

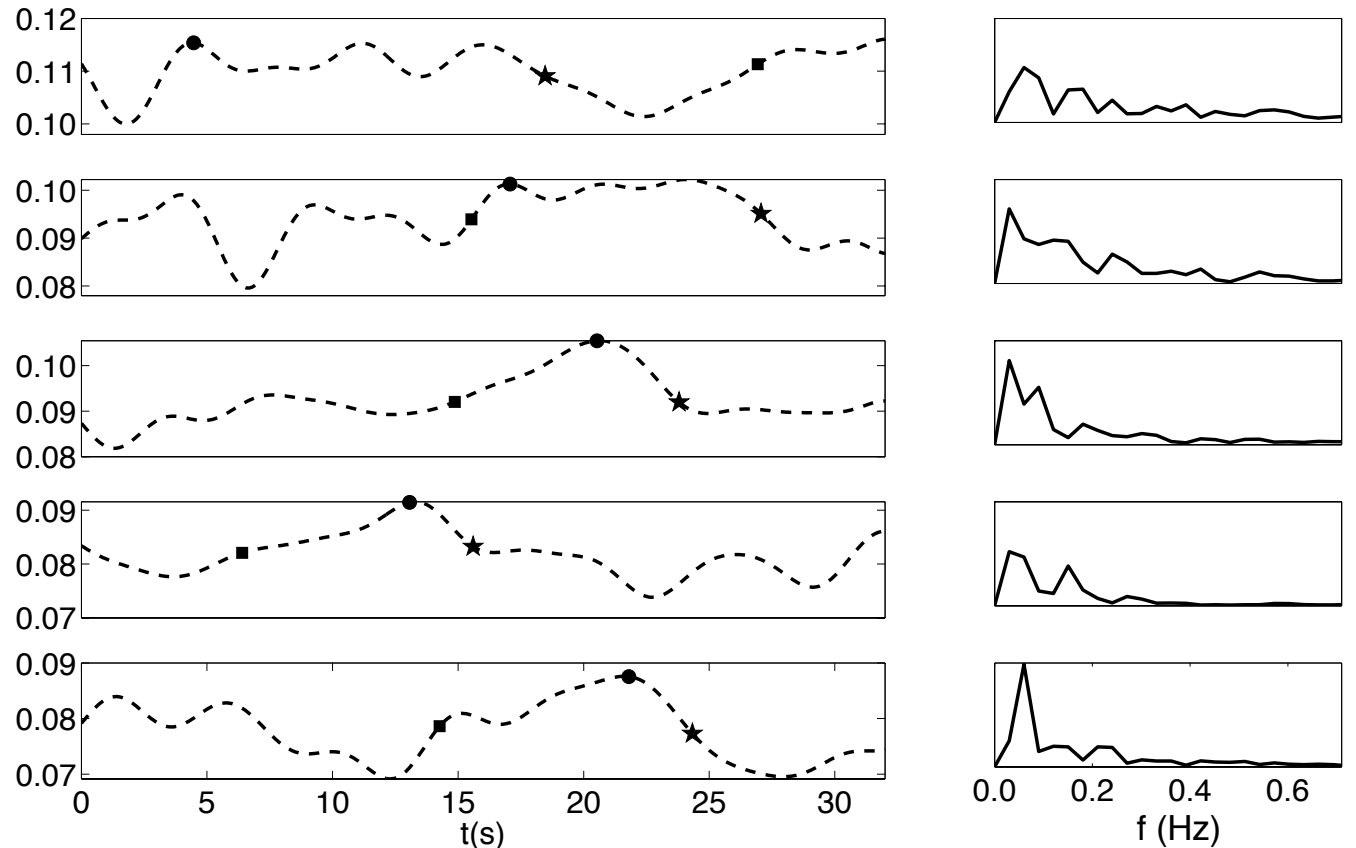

Fig. 4 Left hand side: Time evolutions of the bottom trawl during TRPIV measurements. Right hand side: Corresponding spectral representations. Top to bottom: From measurement plane 1 to measurement plane 5. In each plane, the black circle indicates the highest $y$-position of the trawl during PIV measurements. The black box and the black star are associated with a similar location but correspond to a upward trawl motion and a backward trawl motion respectively. These instants will be future retained to investigate the mean flow field representation (see figures 10, 11, 15 and 16).

extracting the embedded large scale energetic coherent structures. Indeed, the POD decomposition is the optimal decomposition in representing the flow variable energy. Lumley (1967) was the first to apply POD in turbulent flow and proposed to define a coherent structure with POD spatial eigenfunctions that have maximum energy content. Then the application of POD to a given fluctuating velocity field, $\mathbf{u}^{\prime}(\mathbf{X}, t)$, provides a set of mutually orthogonal and deterministic functions acquiring the largest mean square projection of $\mathbf{u}$ '. These functions are the solutions of the following Fredholm equation:

$$
\sum_{j=1}^{N_{c}} \int_{S} R_{i j}\left(\mathbf{X}, \mathbf{X}^{\prime}\right) \phi_{j}^{(n)}\left(\mathbf{X}^{\prime}\right) d \mathbf{X}^{\prime}=\lambda^{(n)} \phi_{i}^{(n)}(\mathbf{X}),
$$

where $R_{i j}\left(\mathbf{X}, \mathbf{X}^{\prime}\right)=\overline{u_{i}^{\prime}(\mathbf{X}) u_{j}^{\prime}\left(\mathbf{X}^{\prime}\right)}$ is the time averaged two-point spatial correlation tensor and an overline represents the time average, $N_{c}$ is the number of the velocity components taken into account and $S$ is the spatial domain. $\phi^{(n)}$ corresponds to the $n^{\text {th }}$ eigenfunction (or mode) of the correlation tensor. The associated eigenvalue $\lambda^{(n)}$ represents the turbulent kinetic energy contained in each mode. The projection of the velocity field onto $\phi^{(n)}$ gives the random projection coefficients, $a^{(n)}(t)$. Each fluctuating velocity component can then be represented by a linear combination of deterministic modes weighted by these random coefficients:

$$
u_{i}^{\prime}(\mathbf{X}, t)=\sum_{n} a^{(n)}(t) \phi_{i}^{(n)}(\mathbf{X})
$$

The previous formulation describes Lumley's original one. In this work the equivalent snapshot version proposed by Sirovich (1987) is used. Briefly, in this case the POD eigenfunctions are now computed from the spatial average time correlation tensor. Deduced temporal modes are mutually orthogonal: $\overline{a^{(n)}(t) a^{(m)}(t)}=\delta_{n m}$, with $\delta$ the Kronecker symbol. $\phi_{i}^{(n)}(\mathbf{X})$ eigenfunctions are determined by the projection of the velocity field onto the temporal eigenfunctions. Similarly, the instantaneous velocity field is expressed with equation 2. Globally, the POD flow decomposition provides 


\begin{tabular}{|l|l|l|l|l|}
\hline Eigenvalue & $\lambda^{\perp}$ & $\lambda^{2}$ & $\lambda^{3}$ & $\lambda^{4}$ \\
\hline \hline Plane 1 & $62.60(98.02 \%)$ & $0.69(1.08 \%)$ & $0.08(0.12 \%)$ & $0.05(0.07 \%)$ \\
\hline Plane 2 & $62.25(97.85 \%)$ & $0.52(0.81 \%)$ & $0.13(0.20 \%)$ & $0.06(0.10 \%)$ \\
\hline Plane 3 & $54.53(97.77 \%)$ & $0.36(0.65 \%)$ & $0.15(0.26 \%)$ & $0.04(0.08 \%)$ \\
\hline Plane 4 & $52.94(97.95 \%)$ & $0.25(0.47 \%)$ & $0.07(0.14 \%)$ & $0.05(0.10 \%)$ \\
\hline Plane 5 & $49.84(97.72 \%)$ & $0.27(0.53 \%)$ & $0.09(0.18 \%)$ & $0.06(0.12 \%)$ \\
\hline
\end{tabular}

Table 1 Energy mode content.

a linear approximation of the energetic dominant feature of the flow which is characterized by a temporal and spatial evolution of each mode.

\subsection{Expansion of POD methodology}

Usually, the POD procedure is carried out from the fluctuating velocity field deduced from the classical Reynolds decomposition. It has been previously applied to many experimental and numerical database to characterize the coherent structures present in turbulent boundary layer flows (see for instance Günther and Von Rohr, 2003; Gurka et al., 2006; Cai et al., 2009; Baltzer and Adrian, 2011 and references therein). As state above, the present flow configuration is fully unsteady. This does not allow the application of classical Reynolds Decomposition because no time averaged flow field can be well estimated. A solution consists in computing the mean flow field in the structureframe of reference. However, this produces a similar mean flow field independently of the trawl motion. We expect that such a solution is not completly satisfactory in the present flow configuration.

A new application of the POD procedure is then proposed to recover the mean flow field around the freely moving porous structure, at each instant. Considering that the mean flow field is the energetic dominant feature of the flow at each instant, it is expected that a linear combination of the first POD modes enables one to identify the dominant mean flow field associated with the motion of the porous fishing structure. To test the effectiveness of this new approach, the following POD application is implemented: a POD is made from the total streamwise velocity component $u$ including the mean and fluctuating flow field. That means computing the eigenfunctions of the correlation tensor given by $R_{u u}\left(\mathbf{X}, \mathbf{X}^{\prime}\right)=\overline{u(\mathbf{X}, t) u\left(\mathbf{X}^{\prime}, t\right)}$ (classic method) or by $R_{u u}\left(t, t^{\prime}\right)=\left\langle u(\mathbf{X}, t) u\left(\mathbf{X}, t^{\prime}\right)\right\rangle$ (snapshot method). This technique is similar to the one previously applied to PIV experimental database of unsteady in-cylinder engine flow (Roudnitzky et al., 2006, Druault and Chaillou, 2007).

\section{Analysis of POD modes and coefficients}

A POD procedure is applied to the whole available instantaneous streamwise velocity component measured in each measurement plane. In each plane, such an application yields $N_{\text {mod }}=N_{t}=500$ POD modes. Note that in each PIV image, instantaneous velocity vectors located under the porous structure during measurements are put to zero value. According to current fully unsteady flow, 500 instantaneous flow fields may not be sufficient to ensure the good statistical convergence of the whole POD modes (Hekmati et al., 2011). However, in the proposed approach, the first POD modes remains always statistically converged, and the PIV measurement errors do not corrupt these first modes (Epps and Techet, 2010).

\subsection{POD energetic content analysis}

As a first analysis, the mode energetic content of POD modes is examined (see table 1). In this table, it is also indicated the percentage of energy contained in each mode. Figure 5 represents the evolution of $\sum_{i=1}^{n} \lambda^{(i)} / \sum_{i=1}^{N_{m o d}} \lambda^{(i)}$ as a function of $n$ for PIV database obtained in plane 3. The first POD eigenvalue contains more than $97 \%$ of the total energy in each measurement plane. This is directly related to velocity database which contains the total velocity field. It is noticeable that the second POD eigenvalue is at least three times higher than the other ones. The first two POD modes seem to extract favorably the dominant energetic phenomena of the flow. 


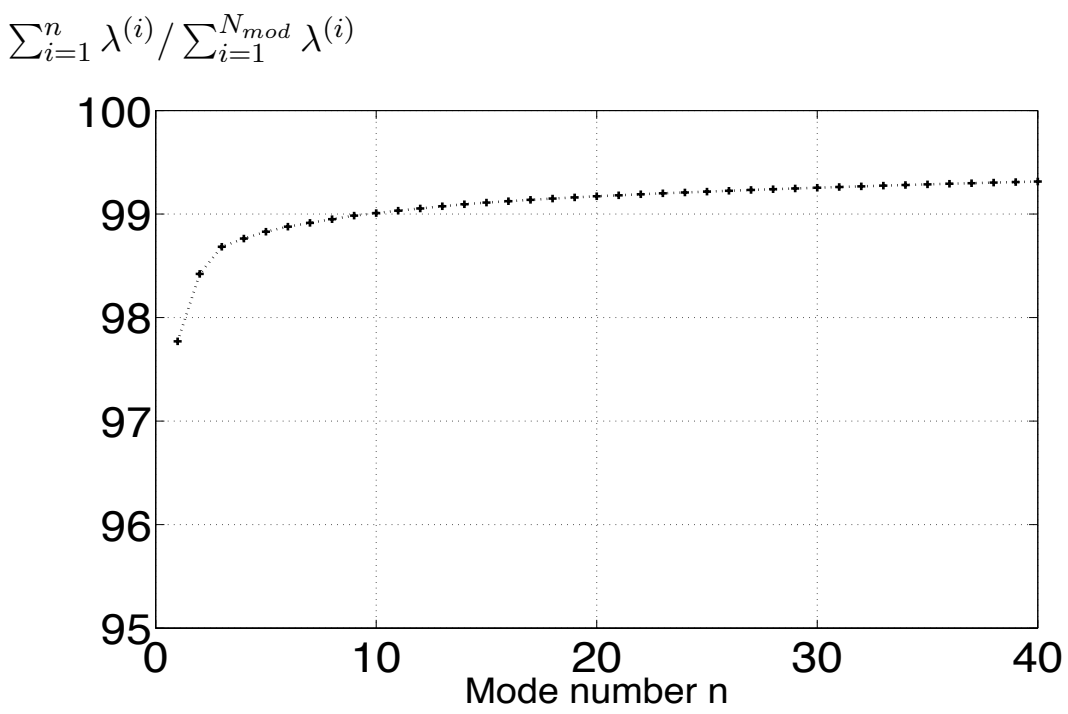

Fig. 5 POD energy convergence. Plane measurement 3.
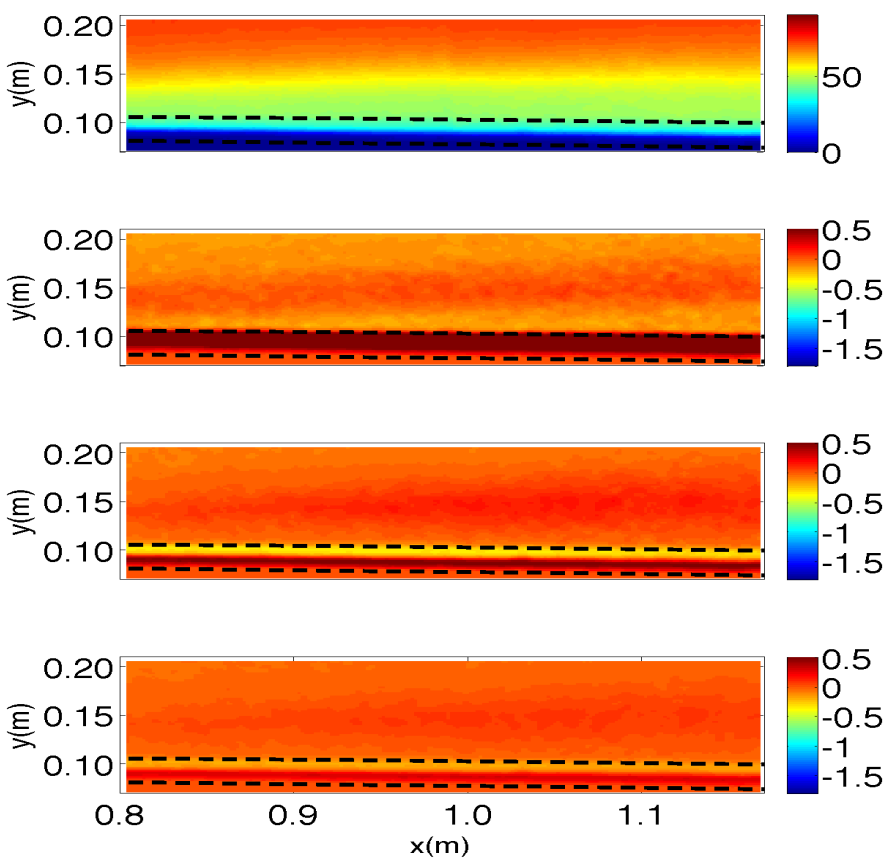

Fig. 6 POD mode analysis. Plane measurement 3. Top to bottom: $\sqrt{\lambda^{(1)}} \Phi^{(1)}(\mathbf{X}) ; \sqrt{\lambda^{(2)}} \Phi^{(2)}(\mathbf{X}), \sqrt{\lambda^{(3)}} \Phi^{(3)}(\mathbf{X}) \cdot \sqrt{\lambda^{(4)}} \Phi^{(4)}(\mathbf{X})$. Dashed line indicates the upper and lower limits of the trawl oscillations during measurements. A same $x$-axis is used for each plot. 
a)

$y(\mathrm{~m})$
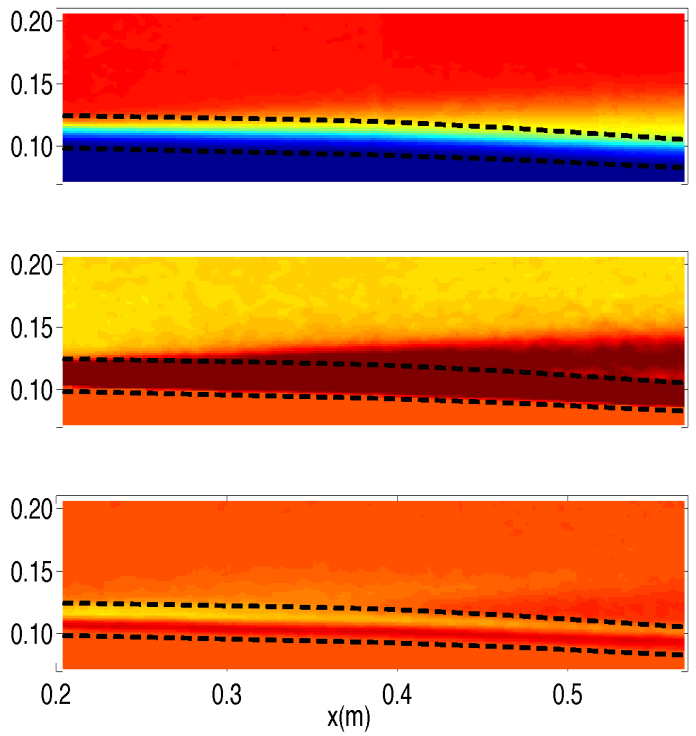

c)

$$
y(\mathrm{~m})
$$
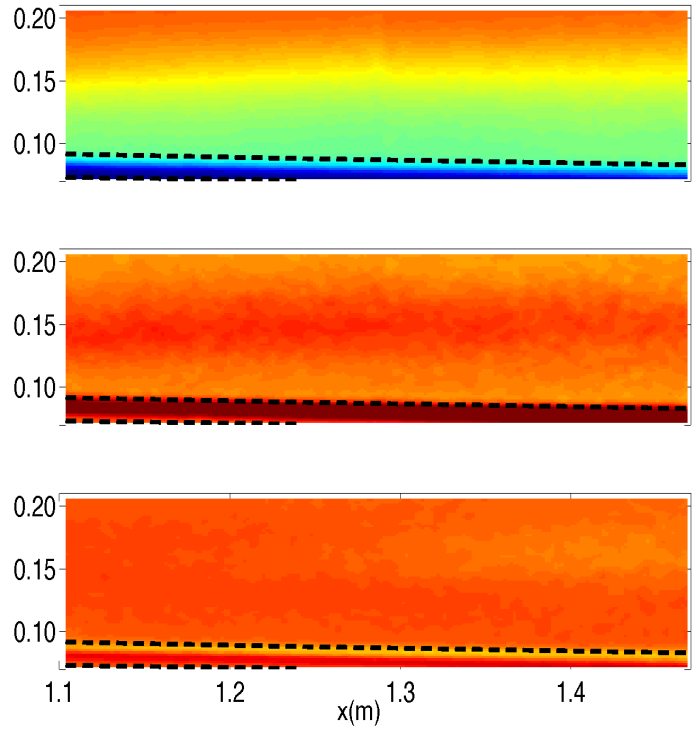

b)

$$
y(\mathrm{~m})
$$
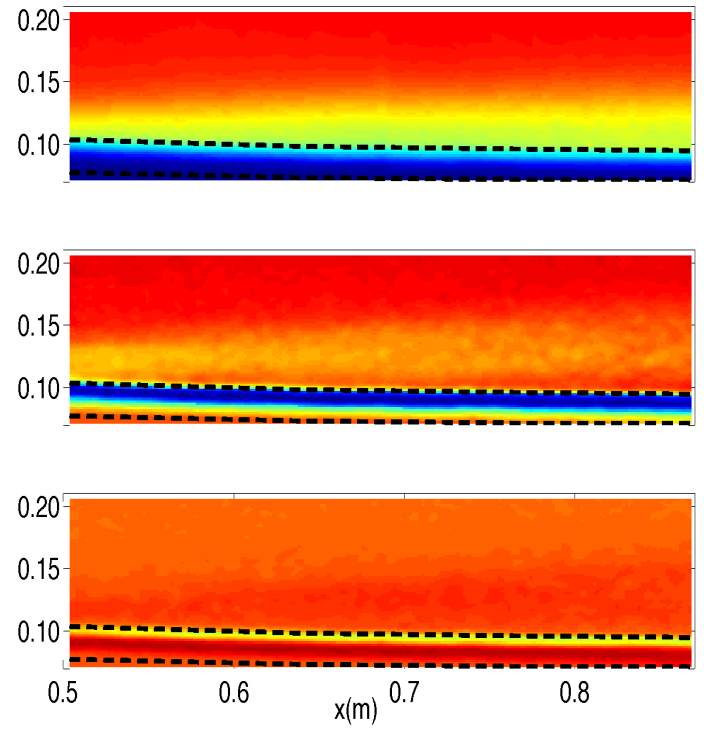

d) $y(\mathrm{~m})$
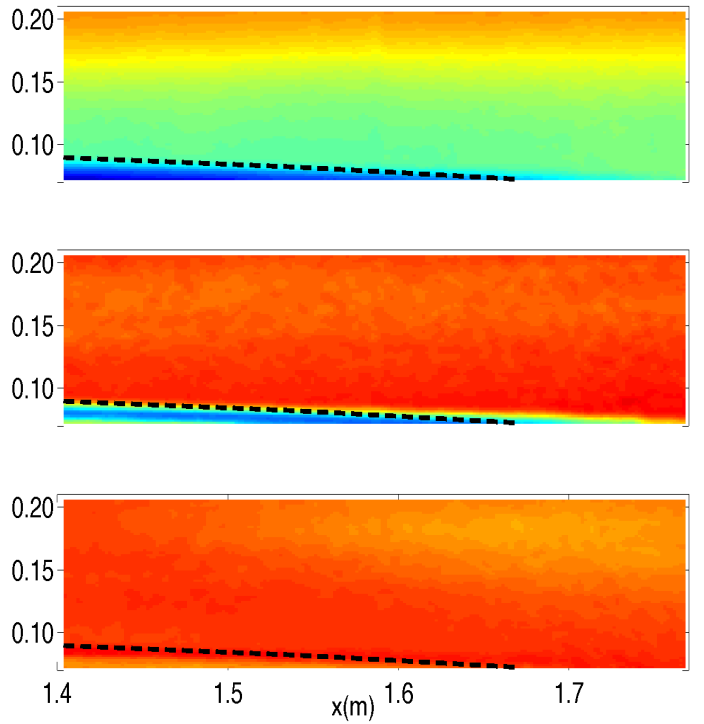

Fig. 7 Spatial POD mode numbers from 1 to 3 (top to bottom). a) PIV plane 1, b) PIV plane 2, c) PIV plane 4 and d) PIV plane 5. Colormaps are similar to the ones given in figure 6 .

\subsection{POD mode shape analysis}

Figure 6 displays the spatial shape of the first four modes $\left(\sqrt{\lambda^{(n)}} \Phi^{(n)}(\mathbf{X}), n=1,4\right)$ obtained in plane 3. The highest and lowest positions of the trawl during measurements are included as black dotted lines. Similar figures are obtained when dealing with the other planes (see figure 7). The first POD mode clearly indicates the signature of the mean boundary layer development. The high values of the first mode shape $\left(\sqrt{\lambda^{(1)}} \Phi^{(1)}(\mathbf{X})\right)$ are directly linked to the high value of the first eigenvalue. Although the values of the second POD modes are much smaller than the ones related 


$$
\begin{aligned}
& a^{(1)}(t), a^{(2)}(t) \\
& a^{(3)}(t), a^{(4)}(t)
\end{aligned}
$$
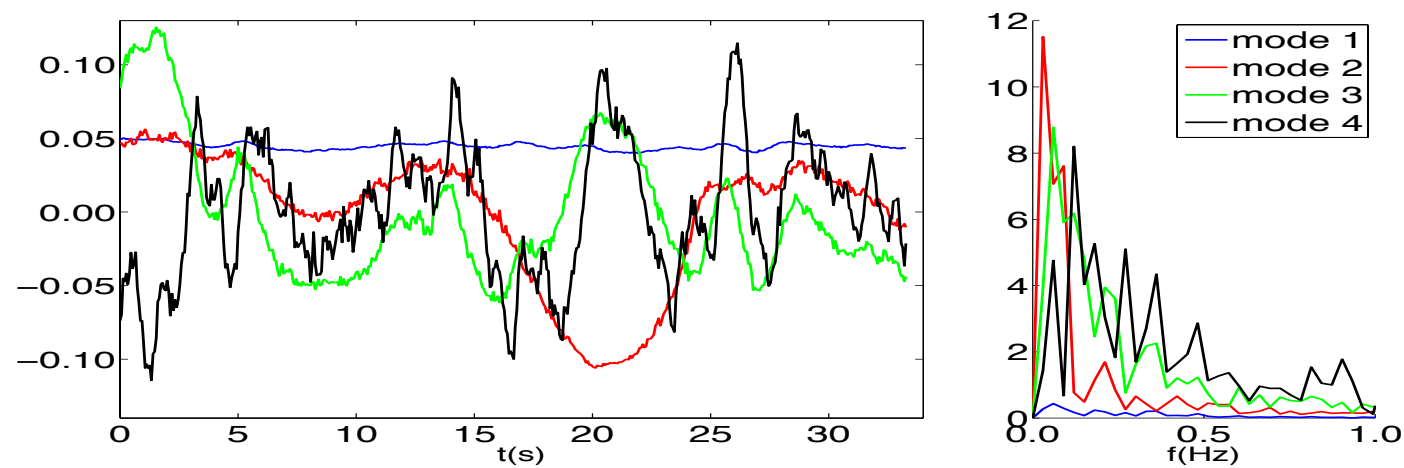

Fig. 8 POD analysis. PIV plane measurement 3. Left hand side: Time evolution of the first four POD coefficients. Right hand side: Corresponding spectra evolution.

to the first POD mode, it is important to note that the changes in these representations are exactly correlated to the trawl motion indicated by black dashed lines. Indeed, these changes are located in the particular zone between the limits of the trawl oscillations. Based on these preliminary results, it seems that a suitable linear combination of the first two POD modes can be interpreted as the the mean flow field (POD mode 1) and the interaction/influence of trawl motion with the mean flow (mode 2).

\subsection{POD temporal coefficient analysis}

Figure 8 represents the time evolution of the first four POD coefficients deduced from PIV database obtained in plane 3. The time evolution of the first POD coefficient is quite similar where a quasi monotonic evolution is observed. The time evolution of the second POD coefficient is very similar to the one associated with the motion of the trawl. Indeed, in each measurement plane, the same tendencies are observed (see Figure 9). In this last figure, the second $P O D$ coefficient computed in each other measurement planes is plotted versus time and it is superimposed onto the time evolution of the bottom trawl in each plane. It is then observed that both time evolutions are quite-similar. These results are confirmed when computing the associated spectrum. Indeed, the $2^{\text {nd }}$ POD coefficient has a spectrum which compares quite well with the one computed the time $y$-displacement of the fishing net structure. Conversely, the high order POD coefficients exhibit a more fluctuating behaviour which can be preferentially associated with the fluctuating part of the velocity field. Globally, time-varying first and second coefficients further confirm that they are two complementary modes to represent the time-varying mean flow field.

In summary, the analysis of the first two POD modes clearly reveals two different flow patterns dictating by the mean flow dynamics around the moving structure: mean boundary layer flow field related to the free-stream velocity $U_{\infty}$ and the motion of the fishing net structure.

\section{Results and discussion}

As stated in the introduction, when dealing with flow around a freely moving body, a first problem concerns the determination of the mean flow field. Indeed, due to the porosity of the structure and its random motion, it is not possible to compute the usual mean flow deduced from the Reynolds decomposition. We then propose to determine the streamwise mean flow field by applying POD procedure detailed above. Once this step achieved, boundary layer thicknesses are investigated.

\subsection{Instantaneous mean vector field representation}

Based on the analysis of POD time coefficients and space modes and also on the energetic content of the first POD modes, the first two POD modes are retained to extract the mean flow field at each instant. The mean flow field is 

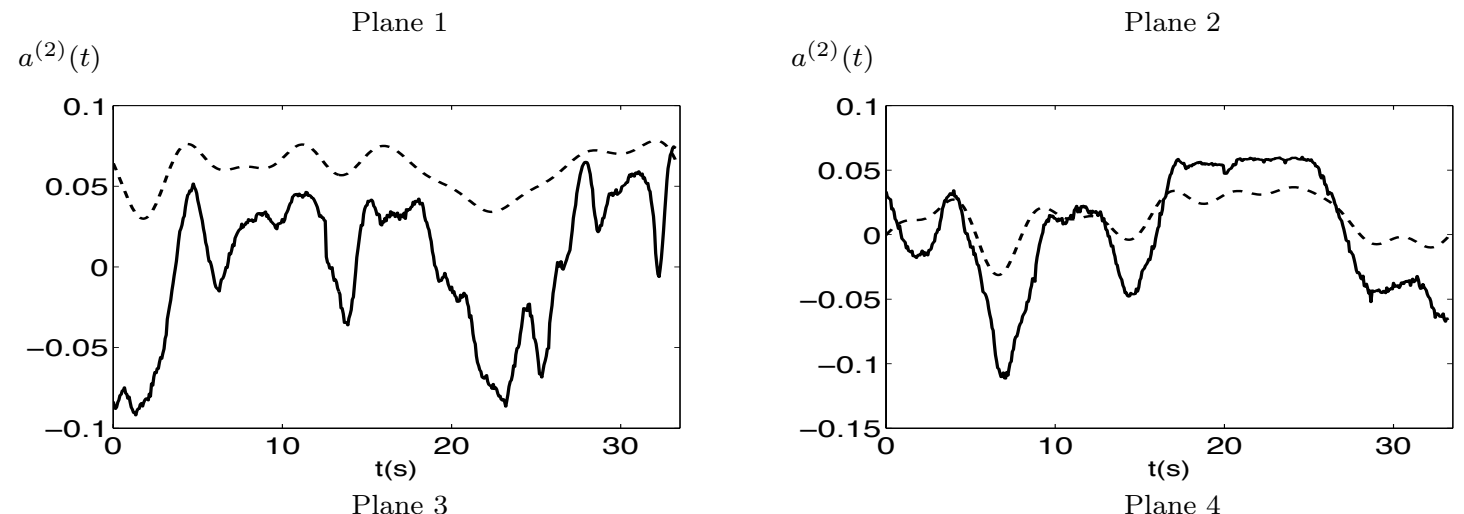

$a^{(2)}(t)$

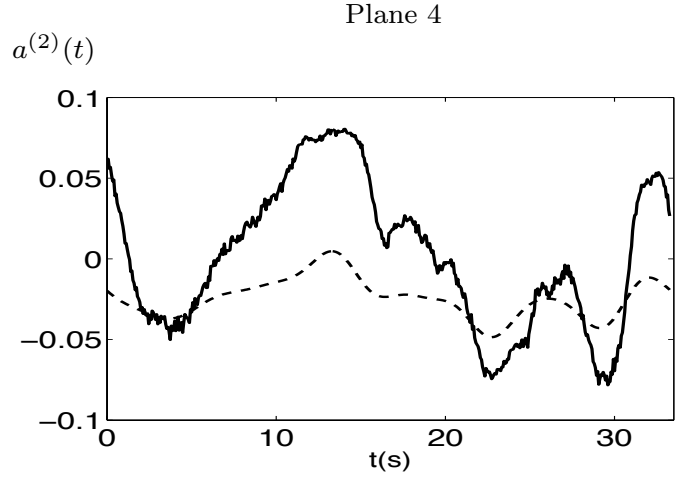

$a^{(2)}(t)$

Plane5

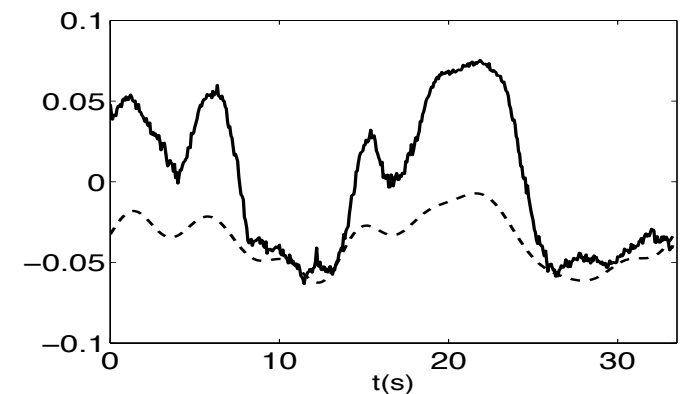

Fig. 9 POD analysis. Time evolution of the second $P O D$ coefficient (black line) superimposed onto the time evolution of the $y$-displacement of the bottom trawl (dotted line). Note that $y$-ticks are associated with POD coefficient time evolutions and they do not correspond to any physical lengths for $y$-displacements (see figure 4 for real $y$-displacement representations)

computed as follows:

$$
u_{m}(t, \mathbf{X})=\sum_{n=1}^{2} a^{(n)}(t) \Phi^{(n)}(\mathbf{X})
$$

Notice that 3 POD modes have also been tested to recover the mean flow field. But any instantaneous mean flow field and also further analysis can not enable to state that this third POD mode has to be included to compute the mean flow field. Indeed, very little difference is observed when dealing with the first 3 modes. Observed discrepancies between mean flow computed from the first two POD modes and the first 3 ones are less than $1 \%$.

Figures 10 and 11 provide some illustrations of the mean flow field computed from equations 3 for each available PIV database. In these figures, for clarity, only one of the ten PIV velocity vectors along the streamwise direction are plotted. These mean flow fields are obtained at selected instants which correspond to the highest amplitude of the trawl oscillations and to the time average value of the $y$-displacement associated with an upward or backward motion respectively (figure 4 ). 
As a first sight, it is obvious that the mean flow field is function of the local position of the trawl. The mean flow is then closely linked to the movement of the trawl as well as its curvature and its porosity. It is then emphasized that working in the frame-body of reference can not be satisfactory in this case.

The mean velocity profiles for streamwise location satisfying $x<0.4 \mathrm{~m}$, approach quasi-similar laminar profiles. Conversely, downstream $(x>0.4 \mathrm{~m})$ the boundary layer flow can be divided into two regions. The first one located near the structure surface corresponds to a region where the mean velocity profile increases rapidly. The second more extended one is associated with a low monotonically increase of the velocity profiles. Moreover, for downstream locations (from plane 3 to plane 5), when the trawl motion reaches the crest (highest amplitude), the mean flow profile exhibits in the close vicinity of the net structure higher values than the ones obtained at the other instants. That can be attributed to the local upstream motion of the trawl which carries out the flow field. More analysis will be carried out in $§ 5.3$ when comparing the transverse profiles of streamwise mean velocity at selected streamwise locations.

\subsection{Boundary layer thicknesses}

The boundary layer thickness $\delta_{90}(t, x)$ computed for the streamwise $x$ position at instant $t$, is defined as the value of $y$ at which $u_{m}(t, x)=0.9 U_{\infty}$. Note that usually $\delta_{99}$ is preferentially computed. However, in the present study the mean flow varies as a function of the random position of the moving trawl and the implementation of POD to recover the mean flow field may not provide satisfactory $\delta_{99}$ results. The displacement thickness $\delta^{*}(t, x)$ and momentum thickness $\theta$ are computed from the following integral equations :

$$
\begin{gathered}
\delta^{*}(t, x)=\int_{0}^{\infty}\left(1-\frac{u_{m}(t, y, x)}{U_{\infty}}\right) d y \\
\theta(t, x)=\int_{0}^{\infty} \frac{u_{m}(t, y, x)}{U_{\infty}}\left(1-\frac{u_{m}(t, y, x)}{U_{\infty}}\right) d y
\end{gathered}
$$

These boundary layer thicknesses are computed from the origin that corresponds to the $y$-position of the porous structure. This is then subjected to the error in manually determining the structure. It is expected that the error is of an order of the PIV mesh resolution that is $1.9 \mathrm{~mm}$ in our work. Then when $\delta_{90}$ value corresponds to $5 \mathrm{~cm}$, the error made for such computation is less than $4 \%$.

These variables are associated with the change in mass $\left(\delta^{*}\right)$ and momentum $(\theta)$ fluxes caused by the presence of a viscous boundary layer when compared to an equivalent inviscid flow. Note that the momentum thickness will be further chosen as the outer variable to scale wall-normal distance because it is determined more precisely from experimental data than the other boundary-layer thicknesses (Adrian et al., 2000).

\subsubsection{Instantaneous streamwise evolution of boundary layer thicknesses}

As a first investigation, boundary layer thicknesses $\delta_{90}(t, x), \delta^{*}(t, x)$ and $\theta(t, x)$ are computed from mean flow field deduced from POD application. Figure 12 displays the streamwise evolution of $\delta_{90}$ and $\theta$ determined from $P O D$ results for each available PIV instantaneous mean flow field. The $\mathrm{X}$-axis is limited to $x<1.6 \mathrm{~m}$ because of the motion of trawl which is out of the PIV measurement plane 5 (see figure 3). We can then observe some important discrepancies as a function of the trawl motion, confirming that the boundary layer development depends on trawl oscillations. To evaluate the variations of thicknesses due to the trawl motion, the Root Mean Square (RMS) of the boundary layer thicknesses are computed for each streamwise position. Figure 12 represents the streamwise evolution of the RMS values associated with each boundary layer thickness and normalized with the corresponding local boundary layer thickness value. In the following $x$-domain, $0.5 \mathrm{~m}<x<1.6 \mathrm{~m}$, RMS values exhibit a percentage within $15 \%$ and $25 \%$. That result is similar to each boundary layer thickness. The high RMS values obtained in the domain $x<0.5 \mathrm{~m}$ is mainly correlated to the very small value of the thicknesses in this domain where the first development of the TBL is observed. This last result confirms the great disparities of boundary layer thicknesses as a function of trawl oscillations. 


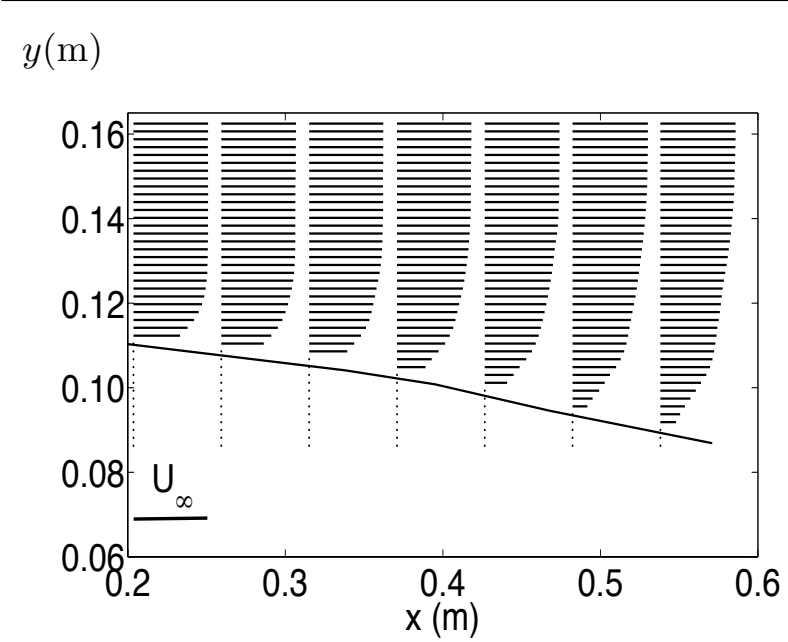

$y(\mathrm{~m})$

$y(\mathrm{~m})$
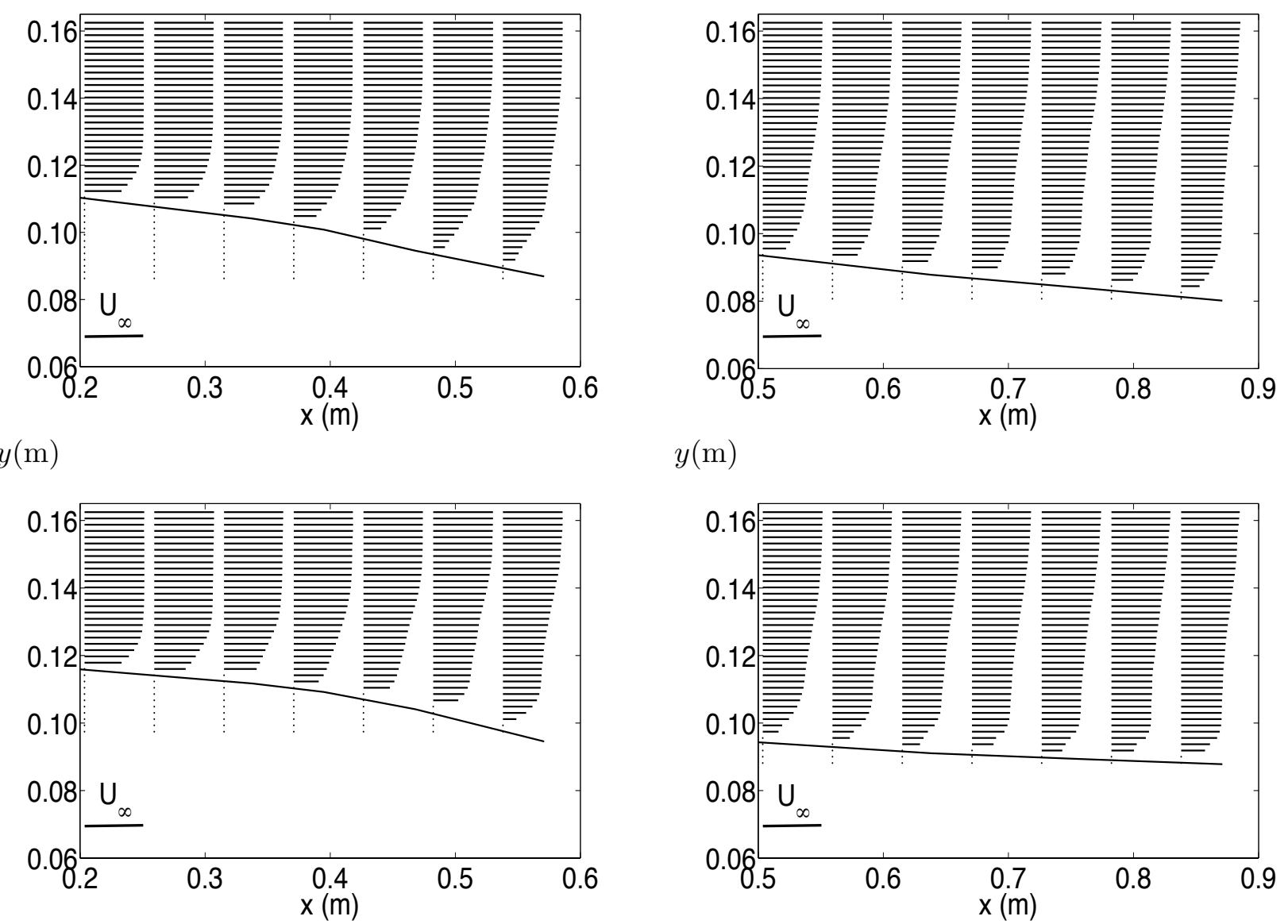

$y(\mathrm{~m})$

$y(\mathrm{~m})$

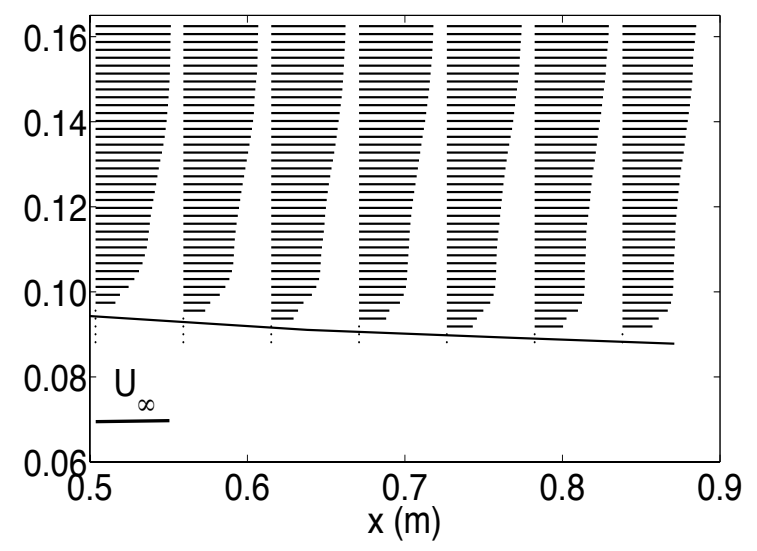
$y(\mathrm{~m})$
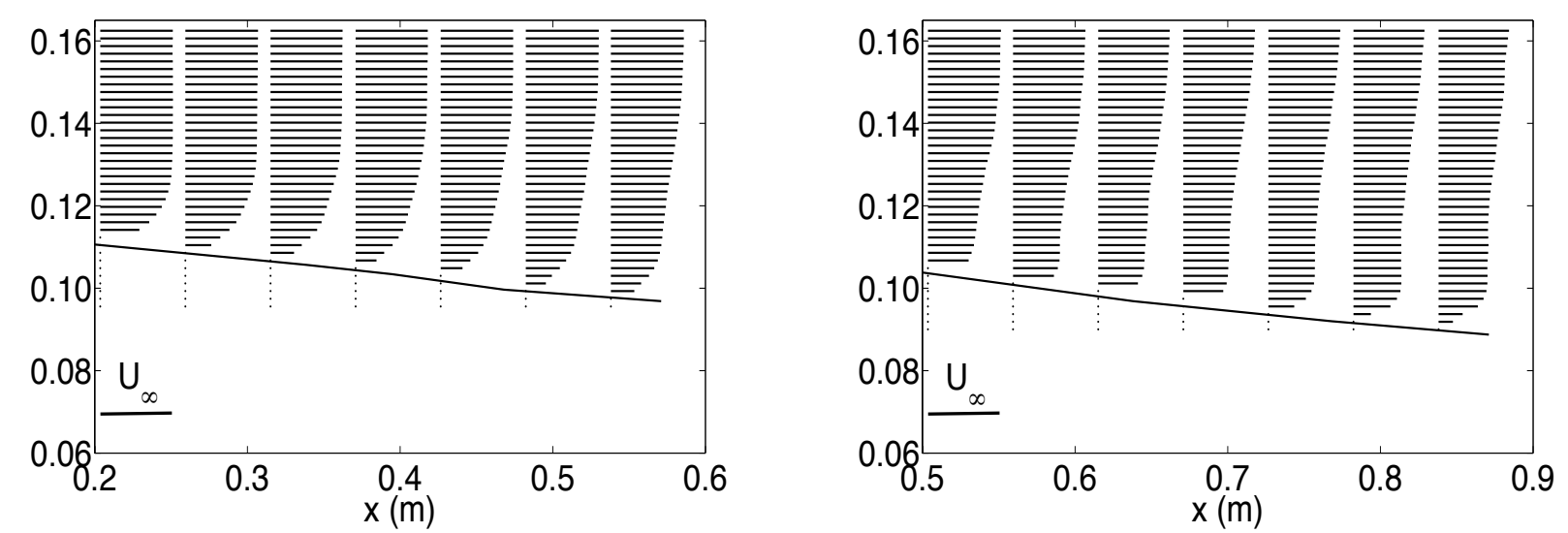

Fig. 10 Left hand side: plane measurement 1. Right hand side: plane measurement 2. Mean velocity vector fields at three different instants. Top to bottom: during the upward motion of the trawl, highest amplitude of the trawl oscillations, during the downward motion of the trawl (details of these instants are given in figure 4). 

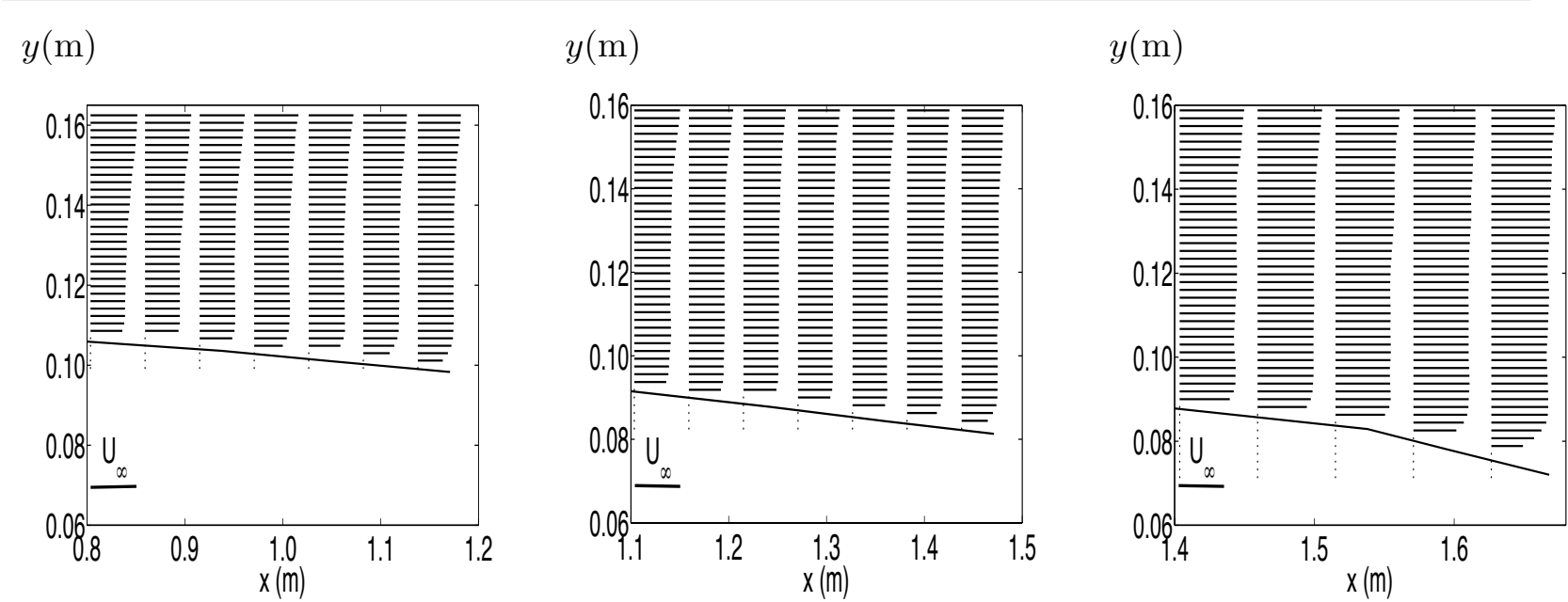

$y(\mathrm{~m})$

$y(\mathrm{~m})$

$y(\mathrm{~m})$
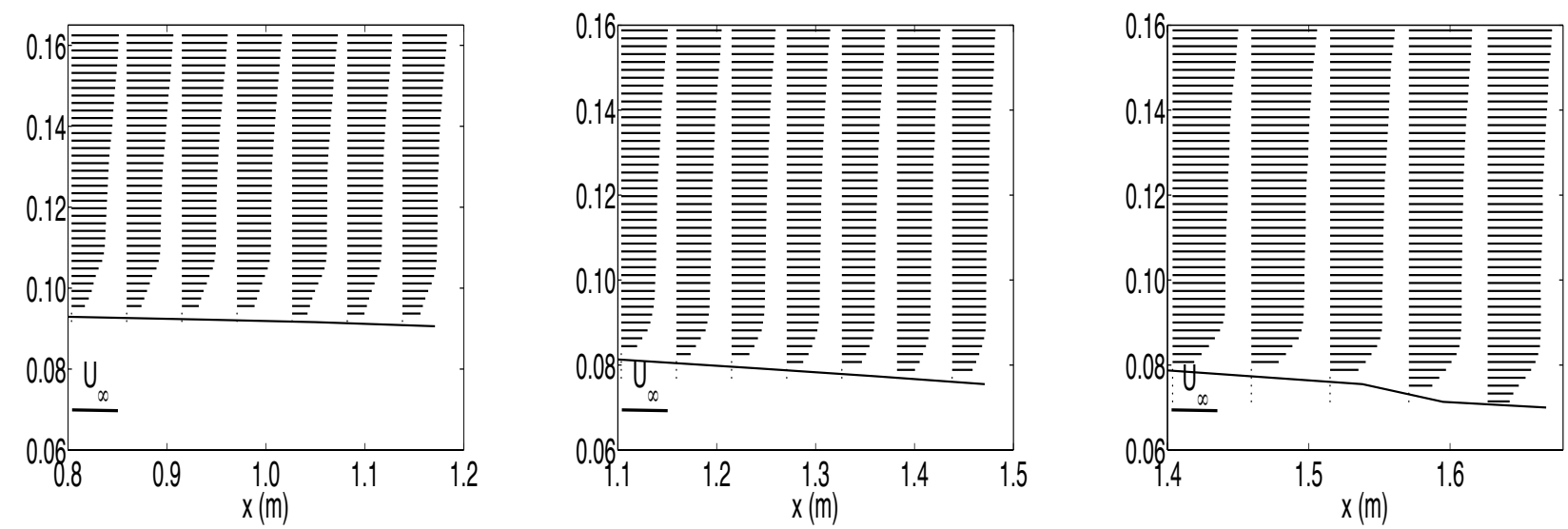

Fig. 11 Left hand side: plane measurement 3. Center: plane measurement 4. Right hand side: plane measurement 5. Mean velocity vector fields at two different instants. Top to bottom: highest amplitude of the trawl oscillations, during the downward motion of the trawl (details of these instants are given in figure 4).

$$
\times 100
$$
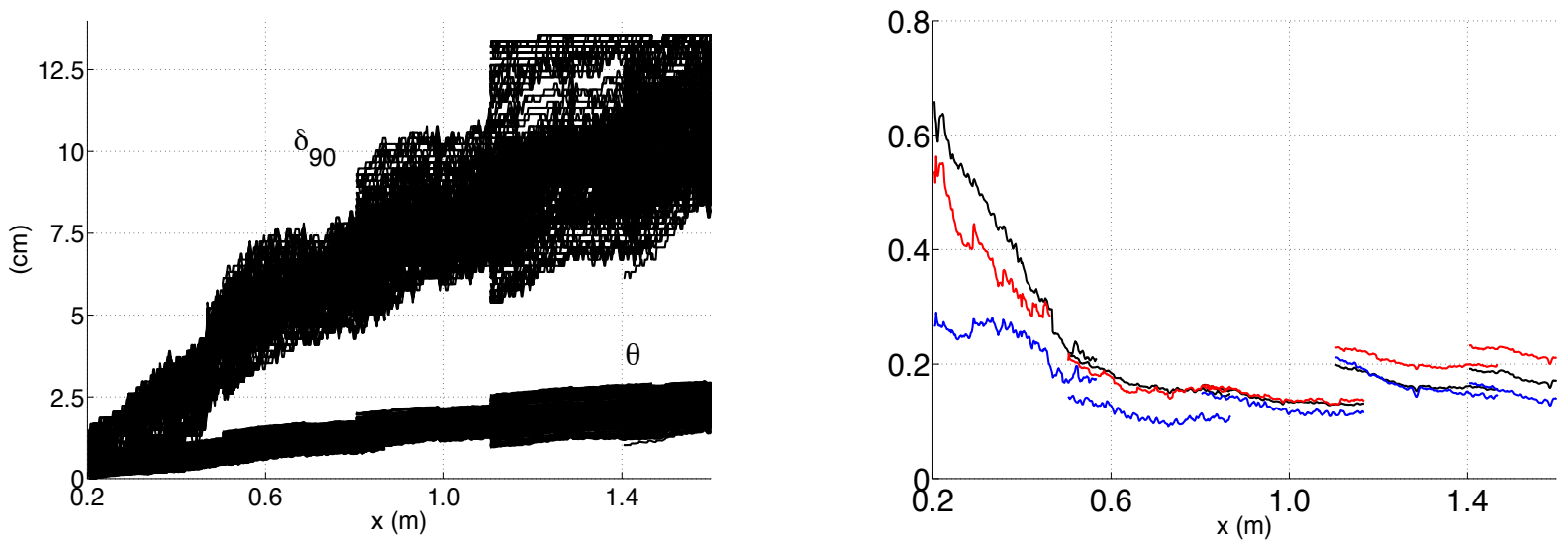

Fig. 12 Left hand side: Streamwise evolution of $\delta_{90}(t)$ and $\theta(t)$ computed from each available instantaneous mean flow field. Right hand side: Plot of the RMS of the boundary layer thicknesses (percentage relative to the local mean value) of boundary thicknesses: $\delta_{90}$ (blue line), $\delta^{*}$ (red line) and $\theta$ (black line). 
$(\mathrm{m})$

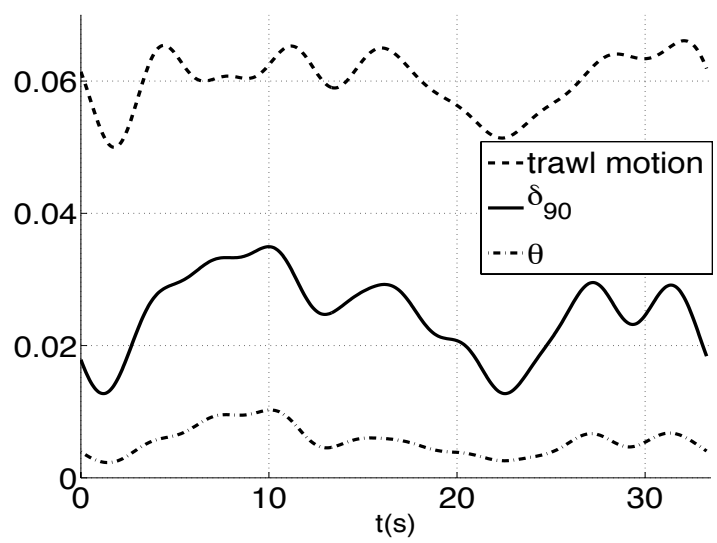

$(\mathrm{m})$

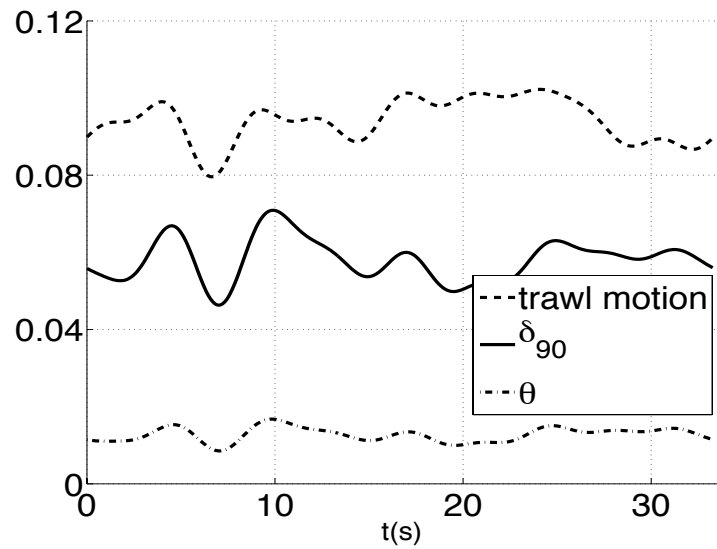

Fig. 13 Time evolution of $\delta_{90}$ and $\theta$ at a fixed streamwise position superimposed onto the associated trawl motion oscillations. Left hand side: Plane 1 . Right hand side: Plane 2. Note that $y$-ticks expressed in meters are associated with $\delta 90$ and $\theta$ representations. They do not correspond to $y$-displacements (see figure 4 for real $y$-displacement representations).

$(\mathrm{m})$

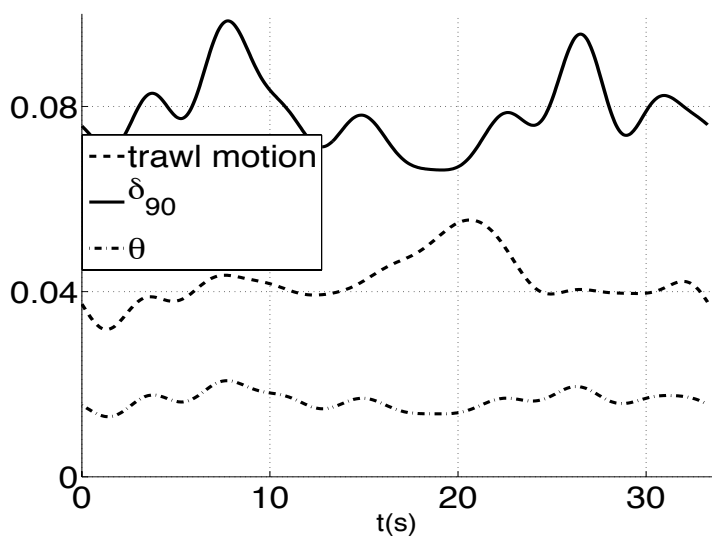

$(\mathrm{m})$

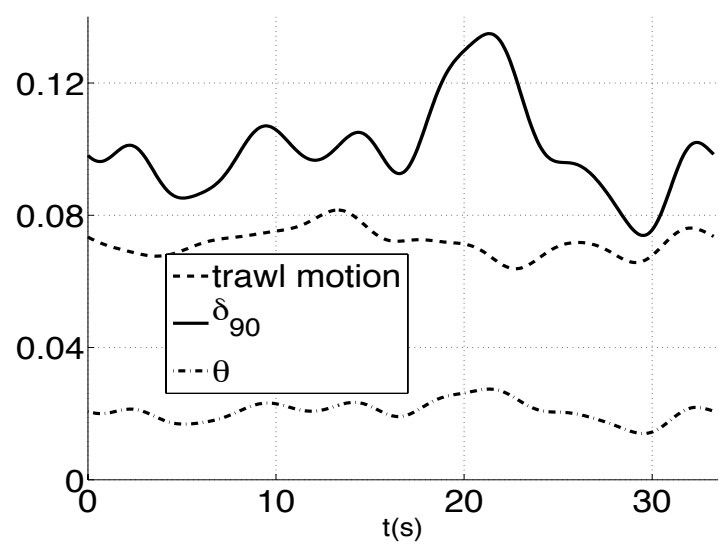

Fig. 14 Time evolution of $\delta_{90}$ and $\theta$ at a fixed streamwise position superimposed onto the associated trawl motion oscillations. Left hand side: Plane 3 . Right hand side: Plane 4 . Note that $y$-ticks expressed in meters are associated with $\delta_{90}$ and $\theta$ representations. They do not correspond to $y$-displacements (see figure 4 for real $y$-displacement representations).

\subsubsection{Boundary layer thickness oscillations}

To investigate the boundary layer development as a function of the trawl oscillations, the time evolution of $\delta_{90}$ and $\theta$ at a fixed streamwise position is regarded (figure 13 and 14). The chosen streamwise positions correspond exactly to the $x$-center of each available PIV measurement plane. Quasi-similar observations are made when using other $x$-location. In these figure, the time evolution of the transverse trawl oscillations is also traced in each PIV plane. Based on planes 1 and 2 results (figure 13), note the great similitude between boundary layer evolutions and trawl oscillations. Furthermore, the range of the whole oscillations of $\delta_{90}$ and the trawl are of the same magnitude order. Such time oscillatory behaviour can be viewed as traveling waves moving in the same direction as the fishing net structure. This is reinforced by the fact that in these planes, the porous structure remains quasi-horizontal allowing the fluid passage to be limited through the structure. In such a case, this flow configuration approaches previous one dealing with boundary layers developing around fish (Anderson et al., 2001) or around the wavy plate (Taneda and Tomonari, 1974) where the same phenomenon was observed. In the other planes, the boundary layer oscillations can not be directly related to the trawl oscillations. The sources of disparities are multiple. Firstly, in the downstream locations, the boundary layer is fully developed and it is also subjected to modifications due to trawl oscillations and the porosity of the net structure. Secondly the amplitude of $\delta_{90}$ time oscillations are higher than the trawl motion 

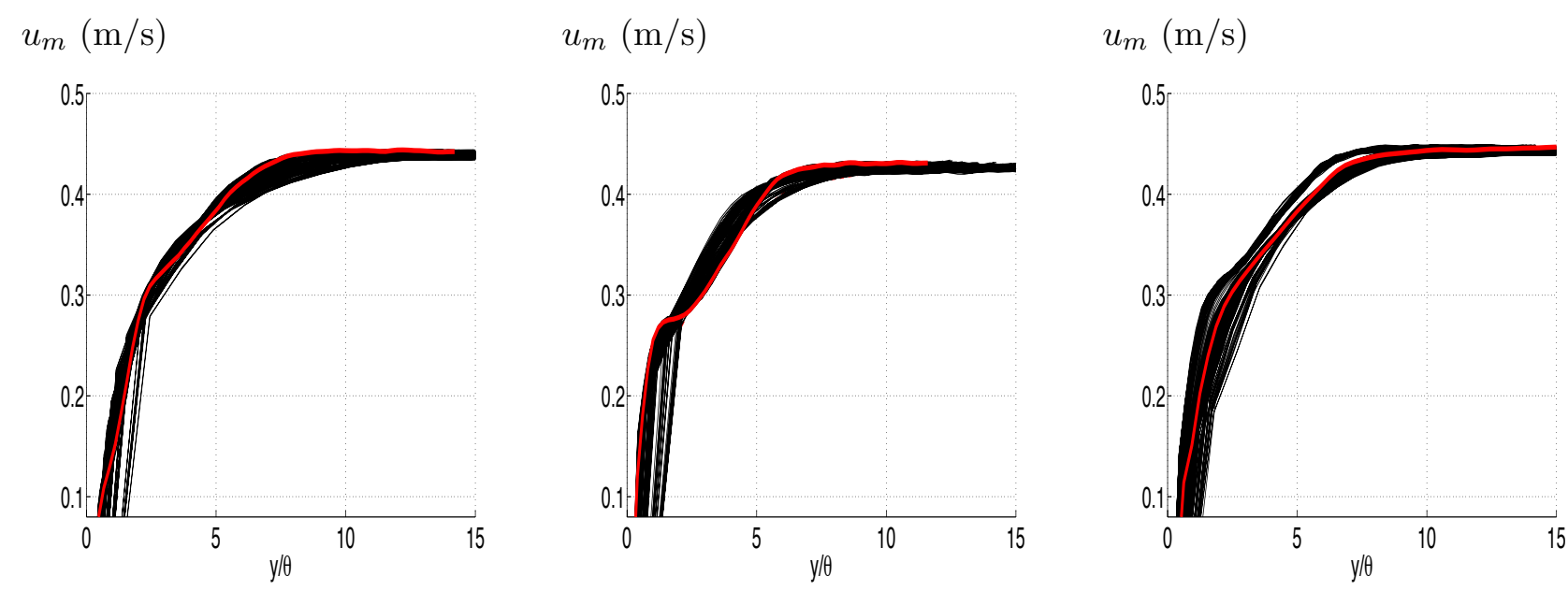

Fig. 15 PIV measurement plane 1. Transverse evolution of the mean flow profiles obtained at selected instants (see figure 4) and at each streamwise position. Red line indicates the mean velocity profile obtained in the $x$-center of the measurement plane. Left hand side: during trawl upward motion. Center: highest amplitude of the trawl motion. Right hand side: during trawl downward motion.

one. Fully developed TBL flow contains large scale vortex organization which differs greatly from the ones that can be observed in the beginning of the development of the TBL. Even if we can see some relationship between trawl oscillations and TBL thickness ones, too many sources of random unsteadiness are present in such a flow that can not enable to well interpret and analyze such behaviour. New investigations about the coherent flow structure dynamics have to be future carried out.

\subsection{Profiles of the mean velocity along the transverse direction}

The transverse evolution of instantaneous mean velocity field (equation 3) is now analyzed. As state before, $\theta$ is retained as the reference length scale because it is more independent of the velocity profile shape than the other thicknesses. The mean streamwise velocity profiles $u_{m}(x, t)$ normalized with $U_{\infty}$ are plotted versus $y / \theta$ (figures 15 and 16). Figure 15 displays the resulting profiles obtained in plane 1 for the three different instants detailed in figure 4. On each plot, the instantaneous velocity profile is represented for each $x$ available position in the measurement plane. Red line indicates the mean velocity profile obtained in the $x$-center of the measurement plane. Figure 16 represents the mean flow profiles at two selected instants and in measurement planes 2,3 and 4 . It appears that when the trawl oscillation rises its highest amplitude in a specific measurement plane, a high velocity gradient near the boundary is observed. Furthermore, theses profiles underline that an increase of the velocity magnitude is obtained near the porous structure. It confirms that even if the fishing net structure is porous, the fluid closest to the structure is most influenced by the motion of the structure though the fluid. This result is quite similar to that of Anderson et al. (2001) who studied the boundary layer of swimming fish. Moreover, further downstream (that is when the trawl motion go downwards), the boundary layer profiles display a relaxation and consequently there is an decrease in the local near-wall velocity gradients. Globally, the shape and the position of the logarithmic region of the time-averaged velocity profile may vary significantly as a result of fishing net surface position.

\section{Conclusions}

A better knowledge of the Turbulent Boundary Layer developing over porous flexible moving structure has been achieved with the aid of Proper Orthogonal Decomposition.

Firstly, the present work demonstrates the feasibility of using the POD procedure to extract an instantaneous mean flow field from unsteady turbulent flow developing around random oscillated structure. It is confirmed that the shape of the mean velocity profiles of such TBL flow depend strongly to multiple parameters such as surface curvature, porosity of the structure, random motion of the structure and also to the upstream history of the boundary layer and the surrounding flow field (far velocity field and inside the porous structure which cannot be accessed in current 


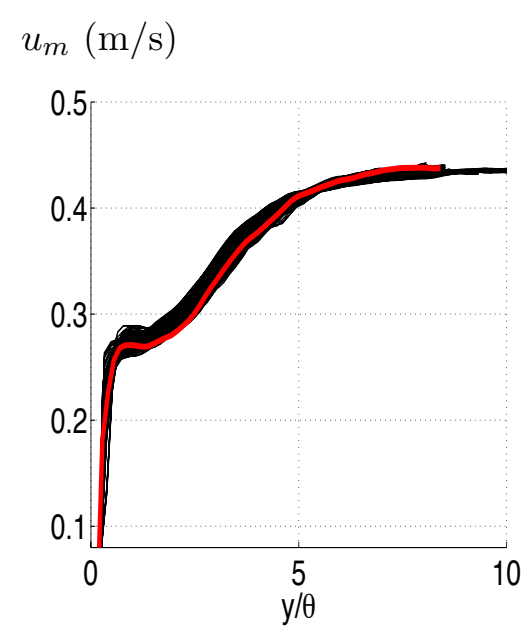

$u_{m}(\mathrm{~m} / \mathrm{s})$

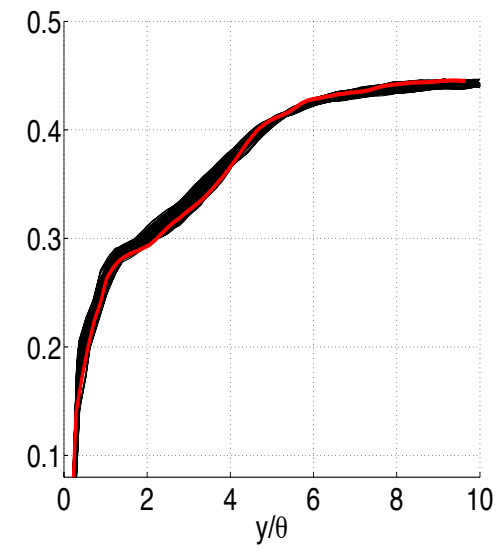

$u_{m}(\mathrm{~m} / \mathrm{s})$

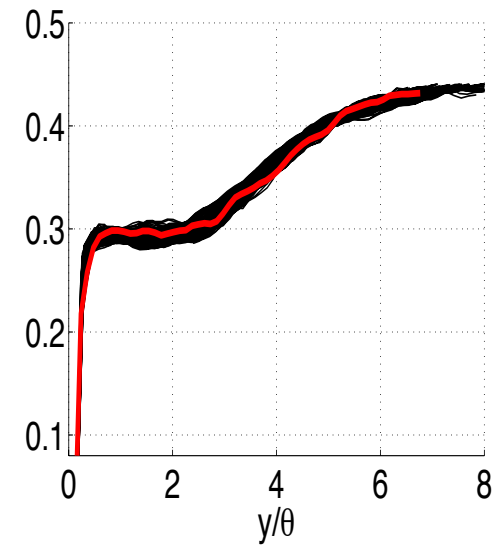

$u_{m}(\mathrm{~m} / \mathrm{s})$

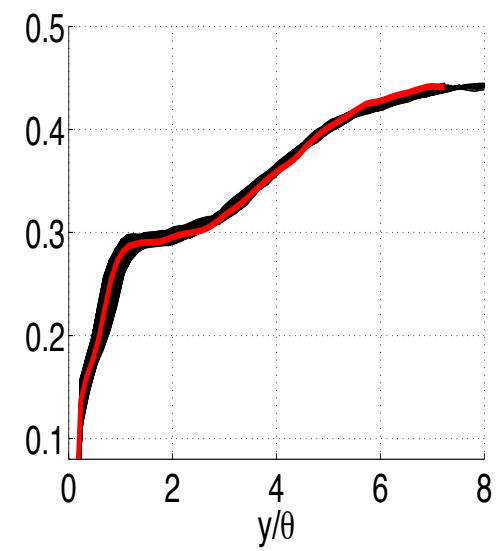

$u_{m}(\mathrm{~m} / \mathrm{s})$

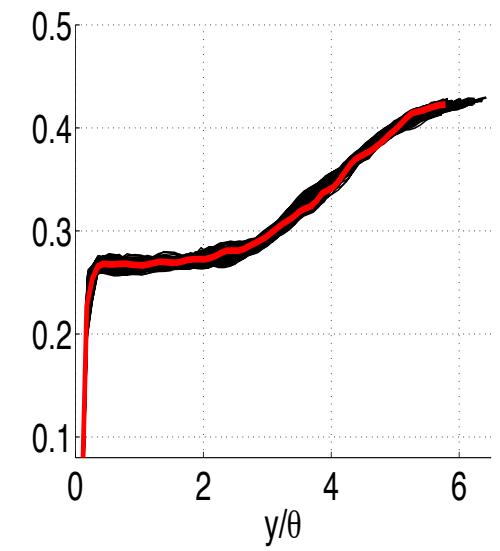

$u_{m}(\mathrm{~m} / \mathrm{s})$

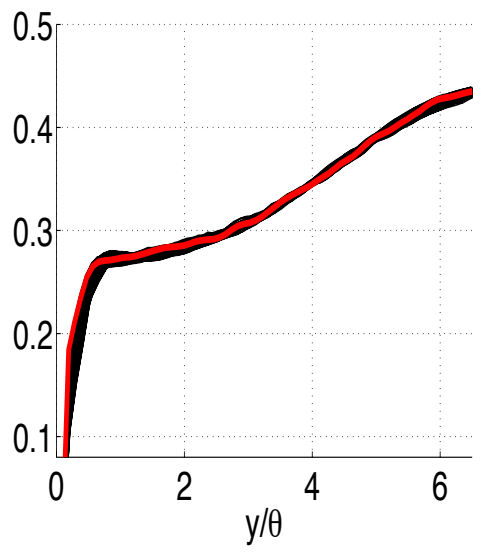

Fig. 16 From left to right: PIV measurement planes from 2 to 4 . Transverse evolution of the mean flow profiles obtained at two instants and at each streamwise position. Red line indicates the mean velocity profile obtained in the $x$-center of the measurement plane. Top: instant corresponding to the highest amplitude of the trawl. Bottom: instant during the trawl downward motion.

measurements). Indeed, it is observed that the transverse curvature and flexibility of the net structure strongly affect the resulting boundary layer development. That reinforces the need to determine instantaneously the mean flow field instead of working in the fixed body-frame of reference.

Such a POD application can find applications in many other unsteady flows associated with fish swimming, arterial flow, insect flighting. As recently outlined by Lauder (2011), when analyzing the hydrodynamics of aquatic animal locomotion, it lacks some post-processing tools or measurement methods aiming at understanding how body motions and induced flows change with time. Current POD application may provide a way for such investigation.

Secondly, the present work provides benchmark PIV data of the unsteady flow developing on fishing net porous structures allowing the advancement of unsteady numerical codes. The results are useful for the validation of the numerical models: they not only give a reasonable description of part of the boundary spatial development, they also present a useful method to access the instantaneous mean flow field. Furthermore, to enhance the overall drag computation of a fishing gear, one has to take into account velocity information in the close vicinity of the porous structure. A future objective is then to introduce these experimental velocity profiles into numerical models aiming at computing the overall drag of a fishing net structure.

\section{Acknowledgements}

The authors would like to gratefully acknowledge the French Ministry of Agriculture and Fisheries and the European Community for their financial support during the HydroPeche project monitored by IFREMER (Institut Français de 
Recherche pour l'Exploitation de la MER). The authors also would like to acknowledge JV. Facq, T. Bacchetti and B. Gaurier for their implication in the experimental work.

\section{References}

1. Adrian RJ, Meinhart CD, Tomkins CD (2000) Vortex organization in the outer regionof the turbulent boundary layer. J. Fluid Mech. 422:1-54.

2. Anderson EJ, McGillis WR, Grosenbaugh MA (2001) The boundary layer of swimming fish. J. Exp. Biol. 204:81-102

3. Baltzer JR, Adrian RJ (2011) Structure, scaling, and synthesis of proper orthogonal decomposition modes of inhomogeneous turbulence. Phys. Fluids 23(1):1-15.

4. Bouhoubeiny E, Germain G, Druault Ph (2011) Time-resolved PIV investigations of the flow field around rigid cod-end net structure. Fisheries Research 108 (2-3):344-355.

5. Brereton GJ (1989) Deduction of skin friction by Clauser technique in unsteady turbulent boundary layers. Exp. Fluids, Vol. 7(6):422-424.

6. Cai WH, Li FC, Zhang HN, Li XB, Yu B, Wei JJ, Kawaguchi Y, Hishida K (2009) Study on the characteristics of turbulent drag-reducing channel flow by particle image velocimetry combining with proper orthogonal decomposition analysis. Phys. Fluids 21(11) 115103:1-12.

7. Carpenter PW, Davies C, Lucey AD (2000) Hydrodynamics and compliant walls: Does the dolphin have a secret? Current Science. 79(6):758-765.

8. Druault Ph, Guibert P, Alizon F (2005) Use of Proper Orthogonal Decomposition for time interpolation from PIV data. Exp. Fluids 39:1009-1023.

9. Druault Ph, Chaillou C (2007) Use of Proper Orthogonal Decomposition for reconstructing the 3D in-cylinder mean-flow field from PIV data. C.R. Mecanique 335(1):42-47.

10. Epps BP, Techet AH (2010) An error threshold criterion for singular value decomposition modes extracted from PIV data. Exp. Fluids 48(2):355-367.

11. Günther A, Von Rohr PR (2003) Large-scale structures in a developed flow over a wavy wall. J. Fluid Mech. 478:257-285.

12. Gurka R, Liberzon A, Hetsroni G (2006) POD of vorticity fields: A method for spatial characterization of coherent structures. Int. J. Heat Fluid Flow 27(3):416:423.

13. Hekmati A, Ricot D, Druault Ph. (2011) About the convergence of POD and EPOD modes computed from CFD simulation. Computers and Fluids 50:60-71.

14. Jimenez J (2004) Turbulent flows over rough walls. Ann. Rev. Fluid Mech. 36: 173-196

15. Kunze S, Brücker C (2011) Flow control over an undulating membrane. Exp. Fluids 50:747-759.

16. Lauder GV (2011) Swimming hydrodynamics: ten questions and the technical approaches needed to resolve them. Exp. Fluids 51:23-35.

17. Lumley JL (1967) The structure of inhomogeneous turbulent flows. Atm. Turb. and Radio wave Prop. eds Yaglom and Tatarsky pp. 166-178.

18. McHenry MJ, Strother JA, Van Hetten SM (2008) Mechanical filtering by the boundary layer and fluid-structure interaction in the superficial neuromast of the fish lateral system. J. Comp. Physiol. A. 194:795-810.

19. Meyler L (2008) Simulation of net structures hydrodynamic fields. In: Modelling and Simulation', InTech Education and Publishing (eds G. Petrone and G. Cammarata) Chapter 16. pp. 261-282.

20. Paschen M, Winkel HJ, Knuths H (2008) Fluid-structure interactions in pelagic trawls and probable consequences for the selectivity of the fishing gear. Adv. Science Techn. 58:247-256.

21. Paschen M (2009) Analysis of current close to the surface of net structures Workshop DEMaT (Methods for the development and Evaluation of Maritime Technologies, Kinky (Japan)

22. Pichot G, Germain G, Priour D (2009) On the experimental study of the flow around a fishing net. Eur. J. Mech. B/Fluids. 28(1):103-116.

23. Priour D, Herrmann B, O’Neill FG (2009) Modelling axisymmetric cod-ends made of different mesh types. Proc Inst Mech Eng (IMech) Part M: J Eng Marit Environ 223:1-8.

24. Roudnitzky S, Druault Ph, Guibert Ph (2006) Proper Orthogonal Decomposition of in-cylinder engine flow into mean component, coherent structures and random Gaussian fluctuations. J. of Turbulence 7(70):1-19.

25. Schlichting H, Gersten K (2000) Boundary layer theory. 8th revised and enlarged edition. Springer eds.

26. Shen L, Zhang X, Yue DKP, Triantafyllou MS (2003) Turbulent flow over a flexible wall undergoing a streamwise traveling wave motion. J. Fluid Mech. 484:197-221.

27. Siddiqui MHK (2007) Velocity measurements around a freely swimming fish using PIV. Meas. Sc. Techn. 18:96-105.

28. Sirovich L (1987) Turbulence and the dynamics of coherent structures. Part I: Coherent structures. Q. Appl. Math XLV:561571.

29. Taneda S, Tomonari Y (1974) An experiment on the flow around a waving plate. J. Phys. Soc. Japan. 36(6):1683-1689. 